\title{
Emergence of order, self-organization and instabilities in a 1-D array of solitons
}

\author{
Martin Centurion, Ye Pu and Demetri Psaltis \\ California Institute of Technology, Pasadena, California 91125
}

\begin{abstract}
Nonlinear interactions between light and matter can lead to the formation of spatial patterns and self-trapped optical beams. A light pulse propagating in a nonlinear Kerr medium will come to a focus if its power is above a critical value. If the pulse power is much higher than the critical power then the optical beam will break up into multiple filaments [1-3]. Each filament will contain approximately the critical power. We have used carbon disulfide $\left(\mathrm{CS}_{2}\right)$ as the nonlinear material $\left(n_{0}=1.6, n_{2}=3 \times 10^{-15} \mathrm{~cm}^{2} / \mathrm{W}[4]\right)$, which has a critical power of $190 \mathrm{~kW}$ for our laser wavelength of $800 \mathrm{~nm}$. We have used 150 -femtosecond pulses with a maximum energy of $1 \mathrm{~mJ}$ to generate spatial solitons. Each pulse from the laser is split into pump and probe pulses. The pump pulse is focused into a line (using a cylindrical lens) at the entrance face of a $10 \mathrm{~mm}$ glass cell filled with $\mathrm{CS}_{2}$.

The beam profile of the pump at the exit of the glass cell is imaged onto a CCD camera for different values of the input power (Fig. 1). In the absence of nonlinearity the incident cylindrical beam would diverge, but for high power the beam self-focuses into a thin line (Fig. 1a-c). For $P>$ $100 P_{c r}$, the beam breaks up into individual filaments (Fig. 2d-e). The filaments are seeded by small variations in the input beam and are stable in location and size to small variations in the input energy. In other words, the pattern of filaments is repeatable from shot to shot as long as the illuminating beam profile is kept constant. The diameter of the filaments is approximately $12 \mu \mathrm{m}$ and does not change when the energy is increased, while the number of filaments increases with power. For $P>250 P_{c r}$, the output beam profile becomes unrepeatable and the filaments start to fuse into a continuous line (Fig 2f-h). We believe the instability in the beam profile results from interactions between the filaments when the spacing between them becomes small. Part of the energy is scattered out of the central maximum into side lobes. The side lobes are generated through the emission of conical waves from the filaments, during the self-focusing stage [5].
\end{abstract}

(a)

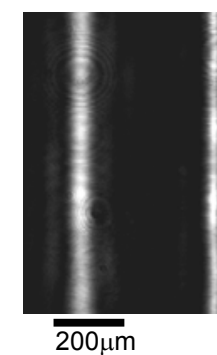

(b)

(c)

(d)

(e)

(f)

(g)

(h)

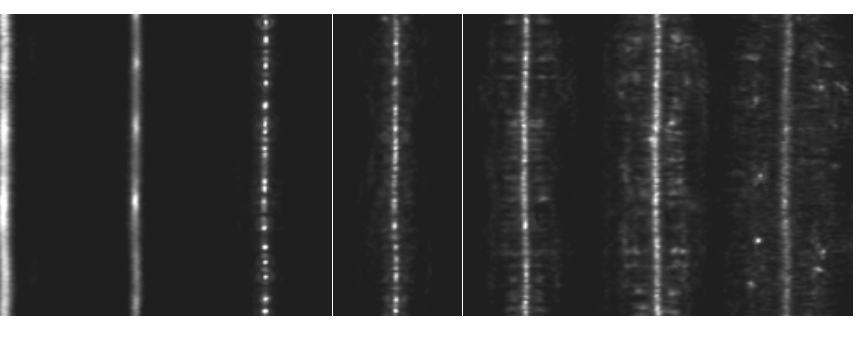

Fig. 1. Beam profile of the pump pulse at the output of the CS2 cell. The power increases form left to right: a) $P=12 P_{c r}$, b) $40 P_{c r}$, c) $80 P_{c r}$, d) $170 P_{c r}$, e) $250 P_{c r}$, f) $390 P_{c r}$, g) $530 P_{c r}$, h) $1200 P_{c r}$.

The beam profile inside the $\mathrm{CS}_{2}$ cell is captured using Femtosecond Time-resolved Optical Polarigraphy (FTOP) [6] in a pump-probe setup. The trajectory of the pump pulse is obtained by numerically combining multiple pump-probe images of the pulse at different positions as it traverses the material. Figure 2 shows the trajectory of the beam obtained for pulses with $P=$ $390 P_{c r}$ (a) and $1200 P_{c r}$ (c), from a distance of $0.5 \mathrm{~cm}$ to $5 \mathrm{~cm}$ from the cell entrance. The 1-D Fourier transforms of the beam profile are calculated and displayed in Figure $2 \mathrm{~b}$ and $2 \mathrm{~d}$ for each 
position along the propagation direction. The pulse with lower power (Fig. 2a) breaks up into filaments at a distance of $2.9 \mathrm{~mm}$ into the material, while the pulse with higher power (Fig. 2c) breaks up at $1.5 \mathrm{~mm}$ from the cell entrance. The Fourier transform in Fig. $2 \mathrm{~b}$ clearly shows how a periodicity emerges during the filamentation process. The filaments are created in a regular array with a $40 \mu \mathrm{m}$ period and propagate undisturbed for several millimetres. If the pulse energy is higher (Fig. 2d) the array of filaments initially forms with a smaller period of $22 \mu \mathrm{m}$, which increases to $33 \mu \mathrm{m}$ as the filaments propagate. After about $5 \mathrm{~mm}$ the sharp peaks visible in the Fourier transform start to fan out and the system transitions to the unstable regime. We attribute the change in the period of the solitons primarily to the interactions between nearby filaments. These interactions cause filament fusion and conical emission, redirecting some of the energy away from the main line of solitons, and causing a rearrangement of the solitons into a sparser grid.

(a)
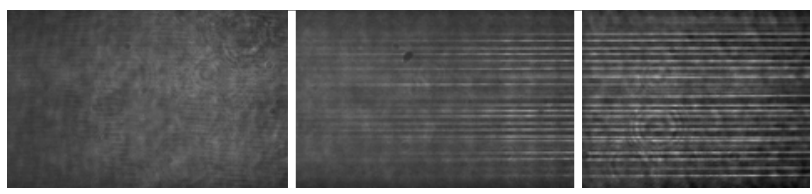

(b)
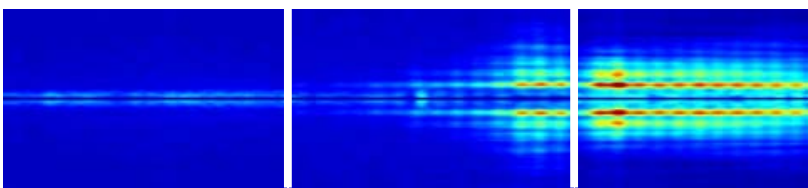

(c)
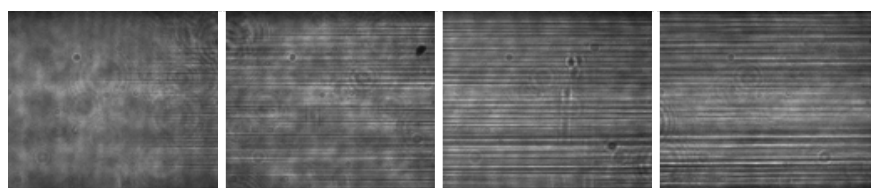

(d)
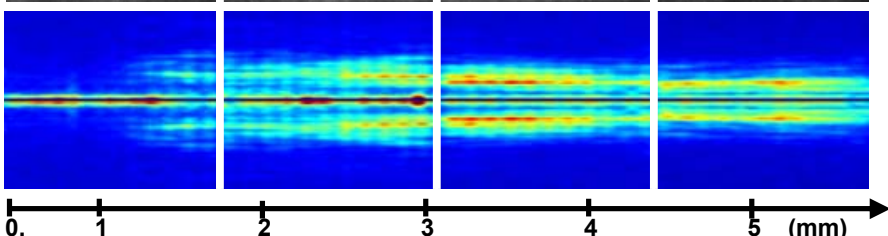

Fig. 2. Pulse trajectories and 1-D Fourier transforms. (a,c): The trajectory of the pulse is reconstructed by digitally adding up the FTOP frames for different positions of the pulse. Each separate image corresponds to frames taken for a fixed position of CCD camera. The camera was moved laterally to capture the beam profile further along inside the cell. The pulse power is $390 P_{c r}$ in (a) and $1200 P_{c r}$ in (c). (b,d) Show the 1-D Fourier transforms of the filamentation patterns in (a) and (c), respectively. The DC component is blocked for clarity.

This work was sponsored by the Engineering Research Centers Program of the National Science Foundation under award EEC-9402726 and the Defense Advanced Research Projects Agency (DARPA) Center for Optofluidic Integration.

\section{References}

1. M. Mlejnek et al., Phys. Rev. Lett. 83, 2938-2941 (1999).

2. L. Berge et al., Phys. Rev. Lett. 92, 225002 (2004).

3. H. Schroeder, S. L. Chin, Opt. Comm. 234, 399-406 (2004).

4. R. A. Ganeev, et al., Appl. Phys. B 78, 433-438 (2004).

5. M. Centurion, Y. Pu, M. Tsang, D. Psaltis, Phys. Rev. A. (to be published).

6. M. Fujimoto et al., Opt. Lett. 24, 850-852 (1999). 